\title{
Wireless Sensing Information Transmission and Storage Technology Research for the Internet of Things
}

\author{
HE Chuan ${ }^{1, \text { a }}$ \\ ${ }^{1}$ School of Electronics and Information Engineering, Jingchu University of Technology, Jingmen \\ 448000, China \\ a hechuan@126.com
}

Keywords: Internet of Things, Wireless Sensing, Information Transmission and Storage

\begin{abstract}
The main objective is to achieve networking integration with a wide range of interconnected information space and depth of the physical world, and wireless sensor networks as a peripheral network of things, it is possible on a larger spatial and temporal scale networking provides active physical perception of things It means the world. Wireless sensor network nodes with limited energy characteristics, and data transmission is the main source of energy consumption in wireless sensor nodes, on the other hand, the presence of Things pressing needs in terms of real time, and data transmission scheduling strategy is the impact of network transmission One of the main factors in the delay, therefore, has important practical significance to study wireless sensor network transmission and storage technologies. In this paper, based on Hadoop HBase is a distributed database that provides real-time read and write, random access, high scalability and high availability storage services. Use HBase to build two storage architectures, metadata are stored within the area sensor data and sensor data.
\end{abstract}

\section{Introduction}

With things in China to promote information technology interface Linhai amount information storage problems. Wireless sensor network end of things as information storage, distribution area more widely, increasing scale, resulting in the rapid growth of information. However, these databases are relational and object model based on complex data storage with high performance capabilities, but the cost and storage systems consume relatively large [1]. In recent years, with the development of cloud computing technology, academia, mass data storage in-depth discussion with the representatives of the Hadoop cluster computing technology and HBase distributed storage system for storing data in columns, HBase is BigTable open source implementation. Single type of sensor data, are single-column data by time distribution and therefore is suitable for information storage HBase wireless sensor networks [2]. According to the characteristics of agricultural sensor wide distribution, the number of many, with Hadoop to build a cluster storage management system, through the HBase distributed storage sensor data. In order to solve data storage problems across the region, the design of two storage architectures, according to the area to build storage cluster, each region has a HBase cluster storage data, design a global data storage area on top of all, to save each area stores information without saving the sensor data collected.

\section{Overview of internet of things}

Things is an Internet-based, traditional telecommunications network and other information carrier, so that all can be implemented interconnection ordinary physical objects individually addressable, providing intelligent network services, it has equipment of ordinary objects, autonomous terminal interconnected and Intelligent universal service important feature. Things break the traditional mode of information technology, the physical world and the world information network to link up the information process extended to the entire space of human activity, using information technology service support all human activities and behavior $p$ [3]. Traditional communication networks (such as the Internet and traditional telecommunication network) to 
achieve interconnection and information exchange between the communication device to form a spatial information. And things put traditional communication network extends to a wider range of the physical world, it is possible to interconnect ordinary physical object, which contains a variety of perception and means of implementation to achieve active interaction with the physical world.

Through networking technology, anything just implanted a tiny electronic tags, you can access the network, while the label stored items related information is also shared. Things take advantage of a variety of sensors to proactively perceive the physical world objects and behavior, and can be a variety of actuators with smart, safe and controlled way to produce a specific behavior of the physical world. We can say that things reflects the spatial information in accordance with our view of things architecture layer structure comprising: an object perception layer, data exchange layer, information integration layer, and application services layer [4-5]. Figure 1 is a schematic view of things architecture.

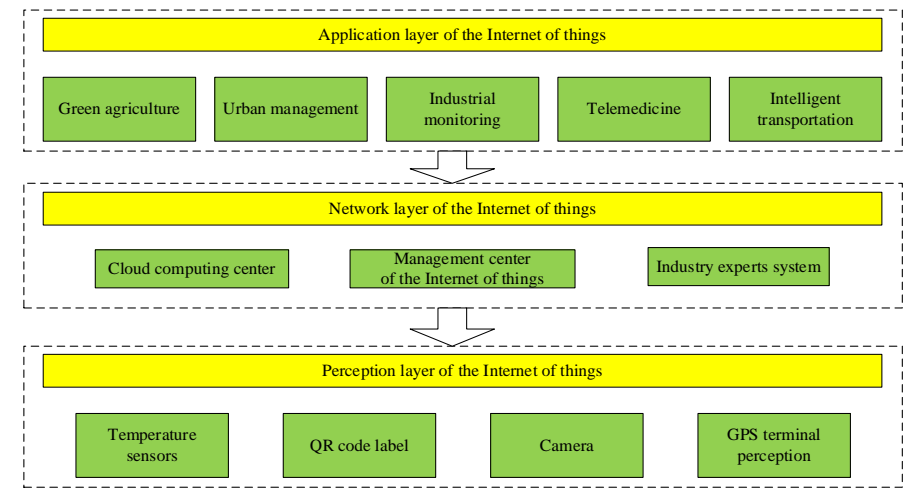

Figure 1.Technology architecture of Internet of things

Object perception layer to achieve the perception of physical objects and data acquisition; data exchange layer provides transparent data transfer capability; information integration layer provides access to the information network of uncertainty completion of the restructuring, cleaning, blending and other processing, integration is relatively accurate conclusions; application service layer information into serving content. Generally believe that the wireless sensor network is an important foundation of Things technology, the wireless sensor network is an important way for effective perception of the physical environment, are key means of Things thorough perception of the physical world.

\section{The analysis of Wireless sensor network system}

Mass deployment of wireless sensor networks based situational awareness with a physical, data processing and wireless sensor node storage, wireless communications and other functions to get the perception of the target within the network coverage of physical properties that can monitor temperature, humidity, light intensity, noise, pressure , gas concentration, and other physical phenomena blessing radiation intensity, then will collect and process transfer information back to the user through a self-organizing wireless communication, for storage or further processing and use [6]. The use of wireless sensor networks for data collection, transmission and processing with a wide range of applications. Figure 2 shows the basic block diagram of a typical wireless sensor networks. Wireless sensor networks are generally mountain wireless sensor nodes, sink nodes (node) and a monitoring center.

A wireless sensor network with one or more sink nodes collect data specifically collected all the sensor nodes, aggregation nodes interact through the gateway data and applications. Wireless sensor networks data storage into internal data storage and external storage applications, external storage issues discussed by the sink node to the data acquisition from sensors. Large-scale sensor networks include two meanings: one is the sensor nodes are distributed over a large geographic area; on the other hand, the sensor nodes are deployed very densely, in a area is not a lot of space, a lot of dense deployments sensor nodes. In practice, widely distributed sensors, large scale, there is a regional deployment, some of the sensors gathered in one area. 


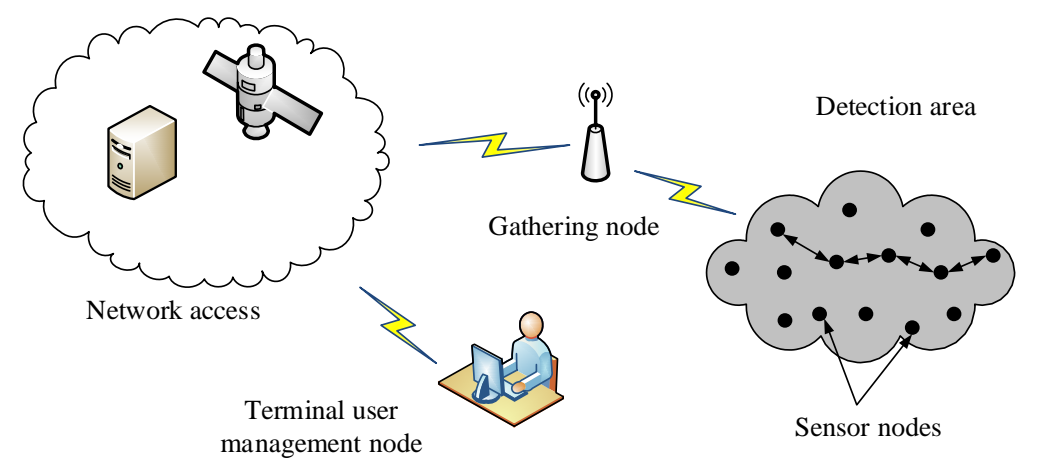

Figure 2.Architecture of wireless sensor network system

\section{Wireless sensor information transmission and storage based on HBase}

HBase is a distributed database, which means that it is used in a cluster of many hundreds of thousands or more servers may run. The result is more complex than running a server installed on an RDBMS. And all the typical problems of distributed computing come into play in the coordination and management of remote processing, etc., lock, data distribution, round-trip delay between the network and the server. Fortunately, HBase use several other mature technologies, such as Apache Hadoop and Apache administrator to resolve these issues. The following figure shows the major components of the HBase architecture. In Figure 3 we can see there is a server HBase master node and a plurality of regions.

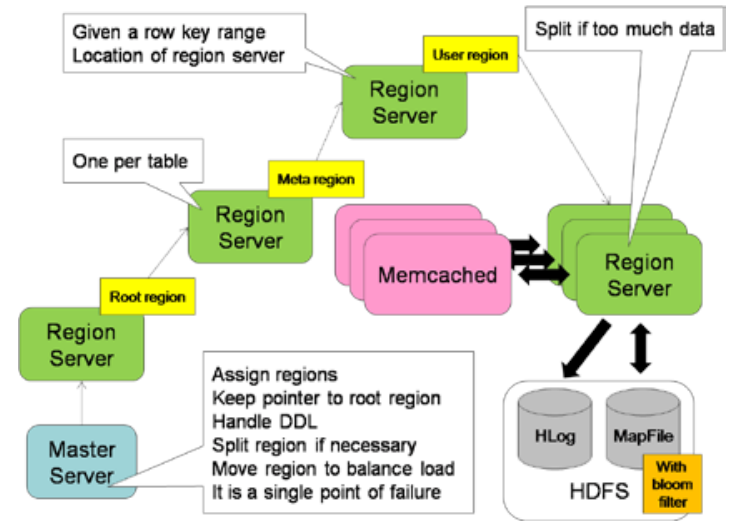

Figure 3. The main architecture components of HBase

(Note that there may be more than one main run HBase settings, there is an active owner.) HBase table is divided into a plurality of areas, each area of the storage line of a series of tables, and the main area servers in multiple regional distribution. HBase is used for data storage, which means that it stores data columns instead of rows. This makes some of the data access patterns are more expensive than the traditional row-oriented relational database system. For example, if there is no data in HBase for a given column family, it just does not store anything, contrast is stored in a relational database must be explicitly null value. In addition, HBase retrieving data, you should only ask for a particular column families need, because there can be thousands of columns in a given row, you just need to make sure you ask your data is actually needed. HBase use administrator (Distributed Coordination Service) to manage regional operations regional servers, and crash recovery server crash area by loading area from the regional servers to other functional areas server. Area contains an in-memory data store (MemStore) and permanent data storage (HFile), and all Regional write-ahead log servers share a reference (cell membrane) is used to store new data has not been saved to permanent storage and server crashes from the region recover. Each region has a specific range of line key, when an area more than the size of a configurable, HBase automatically dividing the area into two sub-regions, which is a key extension of HBase. With the growth of the table, a growing number of regions in the creation and dissemination of the entire cluster. When a 
client requests a particular line key line key or scanned, HBase tell them these key areas exist, then the customer communicate directly with the presence of these regions in the area of server.

This design minimizes the amount of disk seek to find any given line, and disk optimization HBase return data transmission. This is compared to a relational database, which may need to do a lot of disk seeks from the disk before the data transmission, even if there is an index. HDFS Hadoop distributed file system components are distributed, fault-tolerant and scalable file system files to prevent data loss is divided into blocks and spread them across the cluster; it is actually HBase to store data. Strictly speaking, the persistent storage can be any implementation of Hadoop file system API, but usually run Hadoop HBase deploy to HDFS clusters. In fact, when you first download and install HBase on a single machine, it uses the local file system until you change the configuration! Customers and by one of several available HBase API, including local Java API and the rest based interfaces and more an RPC interface.

\section{Wireless sensor data storage architecture}

A wireless sensor network with one or more sink nodes collect data specifically collected all the sensor nodes, aggregation nodes interact through the gateway data and applications. Wireless sensor networks data storage into internal data storage and external storage applications, external storage issues discussed by the sink node to the data acquisition from sensors. Large-scale sensor networks include two meanings: one is the sensor nodes are distributed over a large geographic area; on the other hand, the sensor nodes are deployed very densely, in an area is not a lot of space, a lot of dense deployments sensor nodes. In practice, widely distributed sensors, large scale, there is a regional deployment, some of the sensors gathered in one area. According to the regional distribution of sensor network, data storage architecture design things shown in Figure 4.

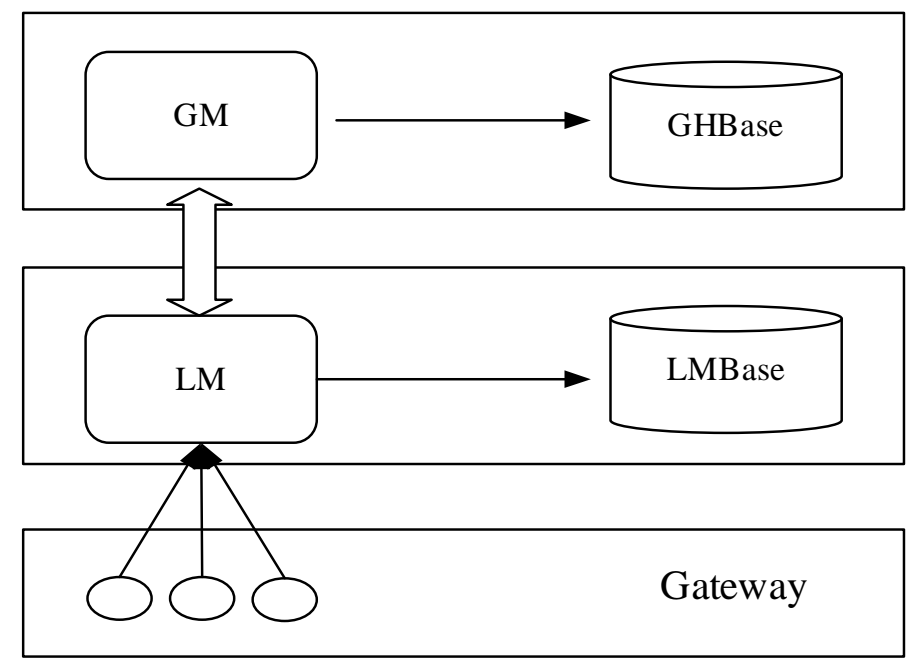

Figure 4. The proposed system architecture

It consists of three levels: (1) the gateway layer: access layer sensor data, different type of sensor has a corresponding gateway to receive and process sensor data. Data collected by the sensor is acquired through a gateway application. (2) LM (Local Manager) layer: Storage Management sensing data, access the sensor from the gateway to collect data, and store the data in real time. (3) GM (Global Manager) level: global information management and record distribution sensor data storage position.

Due to the presence of large-scale regional distribution sensor, if the sensor will store all collected data into a region LHBase (Local HBase) Cluster database will inevitably lead to network resources consumption, the result of a long and time-consuming, and therefore, the data collected is stored in each area region The database server helps to reduce the consumption of network resources, improve real-time store and access data. Therefore, this gateway by region, each region has LHBase used to store real-time sensor data acquisition gateway collected, GHBase (Global HBase) to save the global information, records all LHBase, LM, gateways and sensors and other 
information.

\section{Conclusion}

Wireless sensor network is an important basic technology of things, wireless sensor network is an important way for effective perception of the physical environment, are key means of Things thorough perception of the physical world. The data transmission is the main source of energy consumption in wireless sensor nodes, on the other hand, the presence of Things pressing needs in terms of real time, and data transmission scheduling strategy is one of the main factors affecting the network transmission delay, therefore, for wireless transmission Research sense network transmission and storage technology has important practical significance. This paper provides real-time read and write on HBase, random access, high scalability and high availability storage services. Use HBase to build two storage architectures, metadata are stored within the area sensor data and sensor data.

\section{References}

[1] Li S, Da Xu L, Wang X. Compressed sensing signal and data acquisition in wireless sensor networks and internet of things[J]. Industrial Informatics, IEEE Transactions on, 2013, 9(4): 2177-2186.

[2] Atzori L, Iera A, Morabito G. The internet of things: A survey[J]. Computer networks, 2010, 54(15): 2787-2805.

[3] Zhu Q, Wang R, Chen Q, et al. Iot gateway: Bridgingwireless sensor networks into internet of things[C]//Embedded and Ubiquitous Computing (EUC), 2010 IEEE/IFIP 8th International Conference on. IEEE, 2010: 347-352.

[4] Gubbi J, Buyya R, Marusic S, et al. Internet of Things (IoT): A vision, architectural elements, and future directions[J]. Future Generation Computer Systems, 2013, 29(7): 1645-1660.

[5] Luo J, Chen Y, Tang K, et al. Remote monitoring information system and its applications based on the Internet of Things[C]//BioMedical Information Engineering, 2009. FBIE 2009. International Conference on Future. IEEE, 2009: 482-485.

[6] Mainetti L, Patrono L, Vilei A. Evolution of wireless sensor networks towards the internet of things: A survey[C]//Software, Telecommunications and Computer Networks (SoftCOM), 2011 19th International Conference on. IEEE, 2011: 1-6. 